\title{
Measure of departure from cumulative local symmetry for square contingency tables having ordered categories
}

\author{
Yusuke Saigusa ${ }^{1}$, Tomomasa Takada ${ }^{2}$, Aki Ishii ${ }^{2}$, \\ Tomoyuki Nakagawa ${ }^{2}$, Sadao Tomizawa ${ }^{2}$ \\ ${ }^{1}$ Department of Biostatistics, Yokohama City University School of Medicine, Yokohama \\ City, Kanagawa 236-0004, Japan, e-mail: saigusay@yokohama-cu.ac.jp \\ ${ }^{2}$ Department of Information Sciences, Faculty of Science and Technology, Tokyo \\ University of Science, Noda City, Chiba 278-8510, Japan \\ e-mail: t_takad@yahoo.co.jp, a.ishii@rs.tus.ac.jp, t_nakagawa@rs.tus.ac.jp, \\ tomizawa@is.noda.tus.ac.jp
}

\section{SUMmarY}

For square contingency tables with nominal categories, a local symmetry model which indicates the symmetric structure of probabilities for only one pair of symmetric cells is proposed. For ordinal square tables, the present paper proposes (1) another local symmetry model for cumulative probabilities from the upper-right and lower-left corners of the table, and (2) a measure to represent the degree of departure from the proposed model. The measure has the form of a weighted harmonic mean of the diversity index, which includes the Shannon entropy as a special case. Examples are given in which the proposed method is applied to square table data on decayed teeth in Japanese women patients.

Key words: comparison, confidence interval, diversity index, local symmetry, measure, ordered category, weighted harmonic mean

\section{Introduction}

For an $r \times r$ contingency table with the same row and column classifications, let $p_{i j}$ denote the probability that an observation will fall in the $i$-th row and $j$-th column of the table $(i=1, \ldots, r ; j=1, \ldots, r)$. Several symmetry models with respect to the main diagonal are considered. The symmetry (S) model (Bowker, 1948; Bishop et al., 1975, p.282) is defined as

$$
p_{i j}=\psi_{i j} \quad \text { where } \psi_{i j}=\psi_{j i} \quad \text { for all } i \neq j .
$$


The partial symmetry (PS) model (Saigusa et al., 2016) is defined as

$$
p_{i j}=\psi_{i j} \quad \text { where } \psi_{s t}=\psi_{t s} \quad \text { for at least one } s \neq t .
$$

The local symmetry (LS) model (Saigusa et al., 2019b) is defined as

$$
p_{i j}=\psi_{i j} \quad \text { where } \psi_{a b}=\psi_{b a} \quad \text { for only one } a \neq b .
$$

The LS model describes that the cell probability that an observation will fall in the $a$-th row category and the $b$-th $(>a)$ column category is equal to the probability that the observation falls in the $b$-th row category and the $a$-th column category for only one $(a, b)$.

For a square contingency table having ordered categories, some symmetry models for cumulative probabilities from the upper-right and lower-left corners of the table are also considered. Denote the row and column variables by $X$ and $Y$. The cumulative probability is defined as

$$
G_{i j}= \begin{cases}\sum_{s=1}^{i} \sum_{t=j}^{r} p_{s t}=\operatorname{Pr}(X \leqslant i, Y \geqslant j) & \text { when } i<j, \\ \sum_{s=i}^{r} \sum_{t=1}^{j} p_{s t}=\operatorname{Pr}(X \geqslant i, Y \leqslant j) & \text { when } i>j .\end{cases}
$$

Then the $\mathrm{S}$ model can be expressed as

$$
G_{i j}=\Psi_{i j} \quad \text { where } \Psi_{i j}=\Psi_{j i} \quad \text { for all } i \neq j .
$$

The cumulative partial symmetry (CPS) model (Saigusa et al., 2019a) is defined as

$$
G_{i j}=\Psi_{i j} \quad \text { where } \Psi_{s t}=\Psi_{t s} \quad \text { for at least one } s \neq t .
$$

In data analysis, when a model fits the data poorly, we are interested in measuring the degree of departure from the model. For square contingency tables having nominal categories, measures to represent the degrees of departure from the S, PS and LS models have been developed (Tomizawa, 1994; Tomizawa et al., 1998; Saigusa et al., 2016; Saigusa et al., 2019b). These measures are given as forms of weighted arithmetic, geometric and harmonic means of a diversity index (Patil and Taillie, 1982), consisting of cell probabilities. These measures may not be appropriate for ordered tables, in the sense that the values of these measures do not depend on the order of categories. For square contingency tables with ordered categories, 
some measures for structures on the cumulative probabilities have been developed to incorporate information on the order of categories. The measures for the S and CPS models are given as weighted arithmetic and geometric means of the diversity index consisting of the cumulative probabilities. The details of some of the measures introduced in the above are presented in the Appendix.

The purpose of this paper is to propose another local symmetry model for the cumulative probabilities and a weighted harmonic mean measure for the proposed model. The rest of this paper is organized as follows. Section 2 considers the new model and measure. Section 3 gives the approximate confidence interval of the proposed measure. Section 4 provides an analysis of Japanese decayed teeth data in terms of another local symmetry by using the proposed measure. Sections 5 and 6 contain discussion and brief concluding remarks.

\section{Model and measure}

For square contingency tables with ordered categories, we propose the cumulative local symmetry (CLS) model defined by

$$
G_{i j}=\Psi_{i j} \quad \text { where } \Psi_{a b}=\Psi_{b a} \quad \text { for only one } a \neq b \text {. }
$$

The CLS model describes that the probability that an observation will fall in the $a$-th row category or below and the $b$-th $(>a)$ column category or above is equal to the probability that the observation falls in the $b$-th row category or above and the $a$-th column category or below for only one $(a, b)$. Note that the proposed CLS model has a different structure from the LS model.

Assume that $G_{i j}+G_{j i} \neq 0$ for $i<j$ and $G_{k l} \neq G_{l k}$ for $k<l$ except for any one pair $(k, l)$. Then we propose a measure to represent the degree of departure from the CLS model as follows:

$$
\gamma_{C L S}^{(\lambda)}=\frac{\prod_{a=1}^{r-1} \prod_{b=a+1}^{r} \gamma_{a b}^{(\lambda)}}{\sum_{i=1}^{r-1} \sum_{j=i+1}^{r}\left[\left(G_{i j}^{*}+G_{j i}^{*}\right) \prod_{a=1}^{r-1} \prod_{\substack{b=a+1 \\(a, b) \neq(i, j)}}^{r} \gamma_{a b}^{(\lambda)}\right]} \text { for } \lambda>-1
$$


where

$$
\begin{aligned}
& G_{i j}^{*}=\frac{G_{i j}}{\Delta}, \quad \Delta=\sum_{i=1}^{r-1} \sum_{j=i+1}^{r}\left(G_{i j}+G_{j i}\right), \quad G_{i j}^{c}=\frac{G_{i j}}{G_{i j}+G_{j i}}, \\
& \gamma_{i j}^{(\lambda)}= \begin{cases}1-\frac{\lambda 2^{\lambda}}{2^{\lambda}-1} H_{i j}^{(\lambda)} & \text { for } \lambda \neq 0, \\
1-\frac{1}{\log 2} H_{i j}^{(0)} & \text { for } \lambda=0,\end{cases} \\
& H_{i j}^{(\lambda)}= \begin{cases}\frac{1}{\lambda}\left(1-\left(G_{i j}^{c}\right)^{\lambda+1}-\left(G_{j i}^{c}\right)^{\lambda+1}\right) & \text { for } \lambda \neq 0, \\
-G_{i j}^{c} \log G_{i j}^{c}-G_{j i}^{c} \log G_{j i}^{c} & \text { for } \lambda=0 .\end{cases}
\end{aligned}
$$

When the CLS model does not hold, we can see

$$
\gamma_{C L S}^{(\lambda)}=\frac{1}{\sum_{i=1}^{r-1} \sum_{j=i+1}^{r} \frac{G_{i j}^{*}+G_{j i}^{*}}{\gamma_{i j}^{(\lambda)}}} \quad \text { for } \lambda>-1
$$

Therefore, the measure $\gamma_{C L S}^{(\lambda)}$ is expressed as the weighted harmonic mean of the submeasure $\gamma_{i j}^{(\lambda)}$ for the degree of asymmetry on $\left\{G_{i j}^{c}, G_{j i}^{c}\right\}$. For any $\lambda(>-1)$, we have some properties of the measure $\gamma_{C L S}^{(\lambda)}$ as follows:

(i) $\gamma_{C L S}^{(\lambda)}$ must lie between 0 and 1 ,

(ii) $\gamma_{C L S}^{(\lambda)}=0$ if and only if the CLS model holds,

(iii) $\gamma_{C L S}^{(\lambda)}=1$ if and only if the degree of departure from the CLS model is the largest in the sense that $G_{i j}=0$ (then $G_{j i}>0$ ) or $G_{j i}=0$ (then $G_{i j}>0$ ) for all $i<j$, i.e., the cell probabilities in either the upper-right or lower-left triangle of the table are all zeros.

Denote the measures for S and CPS by $\gamma_{S}^{(\lambda)}$ and $\gamma_{C P S}^{(\lambda)}$, respectively (see Appendix). The value of the measure $\gamma_{C L S}^{(\lambda)}$ applied to a table is equal to or less than the values of the existing measures $\gamma_{S}^{(\lambda)}$ and $\gamma_{C P S}^{(\lambda)}$ applied to the table. This is a natural relationship, because the constraint of CLS is weaker than those of S and CPS. 


\section{Approximate confidence interval of measure}

In this section, we shall give the approximate standard error of estimate of $\gamma_{C L S}^{(\lambda)}$ and the large-sample confidence interval of $\gamma_{C L S}^{(\lambda)}$. Assume that a multinomial distribution applies to the $r \times r$ table. Let $n_{i j}$ denote the observed frequency in cell $(i, j)$ of the table $(i=1, \ldots, r ; j=1, \ldots, r)$. The sample version of $\gamma_{C L S}^{(\lambda)}$, denoted by $\hat{\gamma}_{C L S}^{(\lambda)}$, is given by $\gamma_{C L S}^{(\lambda)}$ with $\left\{p_{i j}\right\}$ replaced by $\left\{\hat{p}_{i j}\right\}$, where $\hat{p}_{i j}=n_{i j} / n$ and $n=\sum \sum n_{i j}$. Using the delta method (Agresti, 2013, p.587), we obtain the following theorem.

Theorem 1. $\sqrt{n}\left(\hat{\gamma}_{C L S}^{(\lambda)}-\gamma_{C L S}^{(\lambda)}\right)$ asymptotically $(n \rightarrow \infty)$ has a normal distribution with mean zero and variance $\sigma^{2}\left[\gamma_{C L S}^{(\lambda)}\right]$, where

$$
\sigma^{2}\left[\gamma_{C L S}^{(\lambda)}\right]=\sum_{k=1}^{r} \sum_{\substack{l=1 \\ l \neq k}}^{r} p_{k l}\left(\omega_{k l}^{(\lambda)}\right)^{2}-\left(\sum_{k=1}^{r} \sum_{\substack{l=1 \\ l \neq k}}^{r} p_{k l} \omega_{k l}^{(\lambda)}\right)^{2} \text { for } \lambda>-1,
$$

with

$$
\begin{aligned}
& \omega_{k l}^{(\lambda)}=\frac{\gamma_{C L S}^{(\lambda)}}{\Delta}\left(d_{k l}-\gamma_{C L S}^{(\lambda)} \xi_{k l}^{(\lambda)}\right), \\
& d_{k l}= \begin{cases}\frac{(l-k)(l-k+1)}{2} & \text { for } k<l, \\
\frac{(k-l)(k-l+1)}{2} & \text { for } k>l,\end{cases}
\end{aligned}
$$

$$
\begin{aligned}
& \xi_{k l}^{(\lambda)}= \\
& \begin{cases}\sum_{i=k}^{l-1} \sum_{j=i+1}^{l} \frac{1}{\gamma_{i j}^{(\lambda)}}\left\{1-\frac{2^{\lambda}(\lambda+1)}{2^{\lambda}-1} \frac{G_{j i}^{c}}{\gamma_{i j}^{(\lambda)}}\left(\left(G_{i j}^{c}\right)^{\lambda}-\left(G_{j i}^{c}\right)^{\lambda}\right)\right\} & \text { for } \lambda \neq 0 ; k<l, \\
\sum_{i=l}^{k-1} \sum_{j=i+1}^{k} \frac{1}{\gamma_{i j}^{(\lambda)}}\left\{1-\frac{2^{\lambda}(\lambda+1)}{2^{\lambda}-1} \frac{G_{i j}^{c}}{\gamma_{i j}^{(\lambda)}}\left(\left(G_{j i}^{c}\right)^{\lambda}-\left(G_{i j}^{c}\right)^{\lambda}\right)\right\} & \text { for } \lambda \neq 0 ; k>l, \\
\sum_{i=k}^{l-1} \sum_{j=i+1}^{l} \frac{1}{\gamma_{i j}^{(0)}}\left\{1-\frac{1}{\log 2} \frac{G_{j i}^{c}}{\gamma_{i j}^{(0)}} \log \frac{G_{i j}^{c}}{G_{j i}^{c}}\right\} & \text { for } \lambda=0 ; k<l, \\
\sum_{i=l}^{k-1} \sum_{j=i+1}^{k} \frac{1}{\gamma_{i j}^{(0)}}\left\{1-\frac{1}{\log 2} \frac{G_{i j}^{c}}{\gamma_{i j}^{(0)}} \log \frac{G_{j i}^{c}}{G_{i j}^{c}}\right\} & \text { for } \lambda=0 ; k>l .\end{cases}
\end{aligned}
$$


The asymptotic distribution of $\sqrt{n}\left(\hat{\gamma}_{C L S}^{(\lambda)}-\gamma_{C L S}^{(\lambda)}\right)$ is applicable only when $0<\gamma_{C L S}^{(\lambda)}<1$.

Let $\hat{\sigma}^{2}\left[\gamma_{C L S}^{(\lambda)}\right]$ denote $\sigma^{2}\left[\gamma_{C L S}^{(\lambda)}\right]$ with $\left\{p_{i j}\right\}$ replaced by $\left\{\hat{p}_{i j}\right\}$. According to Theorem 1, $\hat{\sigma}\left[\gamma_{C L S}^{(\lambda)}\right] / \sqrt{n}$ is an estimated approximate standard error of $\hat{\gamma}_{C L S}^{(\lambda)}$, and also $\hat{\gamma}_{C L S}^{(\lambda)} \pm z_{\alpha / 2} \hat{\sigma}\left[\gamma_{C L S}^{(\lambda)}\right] / \sqrt{n}$ is an approximate $100(1-\alpha)$ percent confidence interval of $\gamma_{C L S}^{(\lambda)}$, where $z_{\alpha / 2}$ is the upper $(\alpha / 2)$-th quantile of the standard normal distribution.

\section{Examples}

Table 1, taken from Tomizawa et al. (2006), contains data on decayed teeth in 363 women patients aged from 18 to 39 visiting a dental clinic in Sapporo City, Japan, from 2001 to 2005. Table 1(a) is classified by numbers of decayed teeth in the left side of the patient's mouth and those in the right side. Note that each of these patients has at least one decayed tooth. Table 1 (b) is reclassified by the numbers of decayed teeth in the lower side of the patient's mouth and those in the upper side.

Table 1. Decayed teeth data for 363 women patients aged 18-39 visiting a dental clinic in Sapporo City, Japan, from 2001 to 2005: (a) classified by numbers of decayed teeth in the left and right sides, and (b) classified by those in the upper and lower sides (Tomizawa et al., 2006)

(a) For left and right decayed teeth

\begin{tabular}{ccccc}
\hline & \multicolumn{3}{c}{ Right } & \\
\cline { 2 - 4 } Left & $1-4$ & $5-8$ & $9+$ & Total \\
\hline $1-4$ & 103 & 45 & 1 & 149 \\
$5-8$ & 35 & 84 & 33 & 152 \\
$9+$ & 3 & 17 & 42 & 62 \\
\hline Total & 141 & 146 & 76 & 363 \\
\hline
\end{tabular}

(b) For lower and upper decayed teeth

\begin{tabular}{ccccc}
\hline & \multicolumn{3}{c}{ Upper } & \\
\cline { 2 - 4 } Lower & $1-4$ & $5-8$ & $9+$ & Total \\
\hline $1-4$ & 97 & 62 & 15 & 174 \\
$5-8$ & 20 & 63 & 75 & 158 \\
$9+$ & 2 & 6 & 23 & 31 \\
\hline Total & 119 & 131 & 113 & 363 \\
\hline
\end{tabular}


Table 2. Estimates of $\gamma_{C L S}^{(\lambda)}$, estimated approximate standard errors of $\hat{\gamma}_{C L S}^{(\lambda)}$ and approximate $95 \%$ confidence intervals of $\gamma_{C L S}^{(\lambda)}$, applied to the data in each of Tables $1(\mathrm{a})$ and $1(\mathrm{~b})$

\begin{tabular}{|c|c|c|c|}
\hline \multicolumn{4}{|c|}{ (a) For Table 1(a) } \\
\hline$\lambda$ & $\begin{array}{c}\text { Estimated } \\
\text { measure } \hat{\gamma}_{C L S}^{(\lambda)}\end{array}$ & $\begin{array}{c}\text { Standard } \\
\text { error }\end{array}$ & $\begin{array}{c}\text { Confidence } \\
\text { interval }\end{array}$ \\
\hline-0.5 & 0.006 & 0.013 & $(-0.019,0.031)$ \\
\hline 0 & 0.010 & 0.021 & $(-0.032,0.052)$ \\
\hline 0.5 & 0.013 & 0.027 & $(-0.040,0.065)$ \\
\hline 1 & 0.014 & 0.030 & $(-0.044,0.072)$ \\
\hline 1.5 & 0.014 & 0.030 & $(-0.045,0.074)$ \\
\hline 2 & 0.014 & 0.030 & $(-0.044,0.072)$ \\
\hline
\end{tabular}

\begin{tabular}{|c|c|c|c|}
\hline \multicolumn{4}{|c|}{ (b) For Table 1(b) } \\
\hline$\lambda$ & $\begin{array}{c}\text { Estimated } \\
\text { measure } \hat{\gamma}_{C L S}^{(\lambda)}\end{array}$ & $\begin{array}{c}\text { Standard } \\
\text { error }\end{array}$ & $\begin{array}{c}\text { Confidence } \\
\text { interval }\end{array}$ \\
\hline-0.5 & 0.222 & 0.059 & $(0.106,0.337)$ \\
\hline 0 & 0.345 & 0.083 & $(0.181,0.508)$ \\
\hline 0.5 & 0.409 & 0.093 & $(0.226,0.591)$ \\
\hline 1 & 0.437 & 0.096 & $(0.249,0.625)$ \\
\hline 1.5 & 0.444 & 0.096 & $(0.255,0.632)$ \\
\hline 2 & 0.437 & 0.096 & $(0.249,0.625)$ \\
\hline
\end{tabular}

Table 2 gives the estimated values of the measure $\gamma_{C L S}^{(\lambda)}$ applied to each of the data in Tables 1(a) and 1(b). It also gives the estimated approximate standard errors and the approximate $95 \%$ confidence intervals of the measure. It is seen from Table 2 that the confidence interval of $\gamma_{C L S}^{(\lambda)}$ applied to the data in Table 1(a) includes zero for any $\lambda$. This means that there might be a CLS structure for numbers of decayed teeth in the left and right sides. On the other hand, it is inferred that there is not a CLS structure for numbers of decayed teeth in the upper and lower sides, because the confidence interval of $\gamma_{C L S}^{(\lambda)}$ applied to the data in Table 1(b) does not include zero for any $\lambda$. The confidence interval of the measure $\gamma_{C L S}^{(\lambda)}$ is also useful for comparing the degrees of departure from CLS for different tables. According to Table 2, the degree of departure from CLS for numbers of decayed teeth in the lower and upper sides may be larger than for decayed teeth in the left and right sides.

Table 3 gives the estimated values of the measures $\gamma_{S}^{(\lambda)}$ and $\gamma_{C P S}^{(\lambda)}$ applied to each of the data in Tables 1(a) and 1(b). It can be seen that, for any $\lambda$, 
the estimated value of $\gamma_{C L S}^{(\lambda)}$ applied to each of Tables $1(\mathrm{a})$ and 1(b) is less than those of $\gamma_{S}^{(\lambda)}$ and $\gamma_{C P S}^{(\lambda)}$.

Table 3. Estimates of the measures $\gamma_{C L S}^{(\lambda)}, \gamma_{C P S}^{(\lambda)}$ and $\gamma_{S}^{(\lambda)}$, applied to the data in each of Tables $1(\mathrm{a})$ and $1(\mathrm{~b})$

(a) For Table 1(a)

\begin{tabular}{llcc}
\hline \multicolumn{1}{c}{$\lambda$} & $\hat{\gamma}_{C L S}^{(\lambda)}$ & $\hat{\gamma}_{C P S}^{(\lambda)}$ & $\hat{\gamma}_{S}^{(\lambda)}$ \\
\hline-0.5 & 0.006 & 0.009 & 0.017 \\
0 & 0.010 & 0.015 & 0.028 \\
0.5 & 0.013 & 0.019 & 0.035 \\
1 & 0.014 & 0.021 & 0.038 \\
1.5 & 0.014 & 0.022 & 0.039 \\
2 & 0.014 & 0.021 & 0.038 \\
\hline
\end{tabular}

(b) For Table 1(b)

\begin{tabular}{lccc}
\hline \multicolumn{1}{c}{$\lambda$} & $\hat{\gamma}_{C L S}^{(\lambda)}$ & $\hat{\gamma}_{C P S}^{(\lambda)}$ & $\hat{\gamma}_{S}^{(\lambda)}$ \\
\hline-0.5 & 0.222 & 0.250 & 0.281 \\
0 & 0.345 & 0.380 & 0.418 \\
0.5 & 0.409 & 0.445 & 0.483 \\
1 & 0.437 & 0.473 & 0.510 \\
1.5 & 0.444 & 0.479 & 0.516 \\
2 & 0.437 & 0.473 & 0.510 \\
\hline
\end{tabular}

\section{Discussion}

Consider the $4 \times 4$ artificial cell and cumulative probability tables given in Tables 4 and 5. The cumulative probabilities given in Tables 5(a)-(f) are calculated from the cell probabilities in Tables 4(a)-(f), respectively.

Table 4. Artificial cell probability tables

\begin{tabular}{llll}
\multicolumn{4}{c}{$(\mathrm{a})$} \\
\hline 0.055 & 0.038 & 0.010 & 0.002 \\
0.002 & 0.050 & 0.100 & 0.008 \\
0.040 & 0.400 & 0.050 & 0.030 \\
0.008 & 0.032 & 0.120 & 0.055 \\
\hline
\end{tabular}

(c)

\begin{tabular}{llll}
\hline 0.030 & 0.138 & 0.010 & 0.002 \\
0.002 & 0.025 & 0.100 & 0.008 \\
0.040 & 0.400 & 0.025 & 0.030 \\
0.008 & 0.032 & 0.120 & 0.030 \\
\hline
\end{tabular}

\begin{tabular}{llll}
\multicolumn{4}{c}{$(\mathrm{e})$} \\
\hline 0.003 & 0.238 & 0.010 & 0.002 \\
0.002 & 0.002 & 0.100 & 0.008 \\
0.040 & 0.400 & 0.002 & 0.030 \\
0.008 & 0.032 & 0.120 & 0.003 \\
\hline
\end{tabular}

(b)

\begin{tabular}{cccc}
\multicolumn{4}{c}{$(\mathrm{b})$} \\
\hline 0.040 & 0.088 & 0.010 & 0.002 \\
0.002 & 0.040 & 0.100 & 0.008 \\
0.040 & 0.400 & 0.040 & 0.030 \\
0.008 & 0.032 & 0.120 & 0.040 \\
\hline
\end{tabular}

(d)

\begin{tabular}{llll}
\hline 0.015 & 0.188 & 0.010 & 0.002 \\
0.002 & 0.015 & 0.100 & 0.008 \\
0.040 & 0.400 & 0.015 & 0.030 \\
0.008 & 0.032 & 0.120 & 0.015 \\
\hline
\end{tabular}

\begin{tabular}{llll}
\multicolumn{4}{c}{$(\mathrm{f})$} \\
\hline 0.100 & 0.000 & 0.000 & 0.000 \\
0.002 & 0.099 & 0.000 & 0.000 \\
0.040 & 0.400 & 0.099 & 0.000 \\
0.008 & 0.032 & 0.120 & 0.100 \\
\hline
\end{tabular}


There is a CLS structure in Table 4(a) with $G_{12}=G_{21}=0.05$. For each of Tables 5(b)-(e), the value of $G_{k l}$ equals the value of $G_{k l}$ in Table 5(a) for $k \neq l$ except for $(k, l)=(1,2)$. The ratio $G_{12} / G_{21}$ is 1.0 for Table 4(a), 2.0 for Table 4(b), 3.0 for Table 4(c), 4.0 for Table 4(d), and 5.0 for Table $4(\mathrm{e})$. Table $5(\mathrm{f})$ shows that the cumulative probabilities in the upper-right triangle cells are all zeros for Table 4(f).

Table 5. Artificial cumulative probability tables: the cumulative probabilities in Tables 5(a)-(f) are calculated from Tables 4(a)-(f), respectively

\begin{tabular}{cccc}
\multicolumn{4}{c}{$(\mathrm{a})$} \\
\hline & 0.050 & 0.012 & 0.002 \\
0.050 & & 0.120 & 0.010 \\
0.048 & 0.480 & & 0.040 \\
0.008 & 0.040 & 0.160 & \\
\hline
\end{tabular}

\begin{tabular}{cccc}
\multicolumn{4}{c}{$(\mathrm{b})$} \\
\hline & 0.100 & 0.012 & 0.002 \\
0.050 & & 0.120 & 0.010 \\
0.048 & 0.480 & & 0.040 \\
0.008 & 0.040 & 0.160 & \\
\hline
\end{tabular}

\begin{tabular}{llll}
\multicolumn{4}{c}{$(\mathrm{c})$} \\
\hline & 0.150 & 0.012 & 0.002 \\
0.050 & & 0.120 & 0.010 \\
0.048 & 0.480 & & 0.040 \\
0.008 & 0.040 & 0.160 & \\
\hline
\end{tabular}

\begin{tabular}{llll}
\multicolumn{4}{c}{$(\mathrm{d})$} \\
\hline & 0.200 & 0.012 & 0.002 \\
0.050 & & 0.120 & 0.010 \\
0.048 & 0.480 & & 0.040 \\
0.008 & 0.040 & 0.160 & \\
\hline
\end{tabular}

\begin{tabular}{cccc}
\multicolumn{4}{c}{$(\mathrm{e})$} \\
\hline & 0.250 & 0.012 & 0.002 \\
0.050 & & 0.120 & 0.010 \\
0.048 & 0.480 & & 0.040 \\
0.008 & 0.040 & 0.160 & \\
\hline
\end{tabular}

\begin{tabular}{cccc}
\multicolumn{4}{c}{$(\mathrm{f})$} \\
\hline & 0.000 & 0.000 & 0.000 \\
0.050 & & 0.000 & 0.000 \\
0.048 & 0.480 & & 0.000 \\
0.008 & 0.040 & 0.160 & \\
\hline
\end{tabular}

Table 6 gives the values of the measure $\gamma_{C L S}^{(\lambda)}$ applied to each of Tables 4(a)-(f). We see from Table 6 that (1) the value of $\gamma_{C L S}^{(\lambda)}$ for Table 4(a) is 0 , (2) for any fixed $\lambda$, the value of $\gamma_{C L S}^{(\lambda)}$ increases as the ratio $G_{12} / G_{21}$ increases, and (3) the value of $\gamma_{C L S}^{(\lambda)}$ for Table $4(\mathrm{f})$ is 1 . Therefore, the measure $\gamma_{C L S}^{(\lambda)}$ would be natural to represent the degree of departure from the CLS model.

Consider the artificial cell probability table in Table 7(a), and the modified table interchanging 2 and 3 in categories in Table 7(b). Denote the measure for LS by $\Phi_{L S}^{(\lambda)}$ (see Appendix). Tables $7(\mathrm{c})-(\mathrm{d})$ give the values of the measures $\Phi_{L S}^{(\lambda)}$ and $\gamma_{C L S}^{(\lambda)}$ applied to each of Tables $7(\mathrm{a})-(\mathrm{b})$, respectively. 
Table 6. Values of $\gamma_{C L S}^{(\lambda)}$ for Tables 4(a)-(f)

\begin{tabular}{cccc}
\hline Applied & \multicolumn{3}{c}{$\lambda$} \\
\cline { 2 - 4 } tables & 0 & 0.5 & 1.5 \\
\hline Table 4(a) & 0.000 & 0.000 & 0.000 \\
Table 4(b) & 0.208 & 0.253 & 0.279 \\
Table 4(c) & 0.256 & 0.309 & 0.340 \\
Table 4(d) & 0.278 & 0.334 & 0.366 \\
Table 4(e) & 0.293 & 0.351 & 0.384 \\
Table 4(f) & 1.000 & 1.000 & 1.000 \\
\hline
\end{tabular}

According to Tables $7(\mathrm{c})-(\mathrm{d})$, for any $\lambda$, we can see that the value of $\gamma_{C L S}^{(\lambda)}$ for Table $7(\mathrm{a})$ is different from Table $7(\mathrm{~b})$, while the value of $\Phi_{L S}^{(\lambda)}$ for Table $7(\mathrm{a})$ is the same as Table $7(\mathrm{~b})$. This is because the measure $\gamma_{C L S}^{(\lambda)}$ depends on the order of categories, unlike $\Phi_{L S}^{(\lambda)}$.

Table 7. (a) Artificial cell probability table and (b) modified table interchanging 2 and 3 in categories, and (c)-(d) the values of $\Phi_{L S}^{(\lambda)}$ and $\gamma_{C L S}^{(\lambda)}$ applied to the data in each of Tables $7(a)-(b)$

\begin{tabular}{llll}
\multicolumn{4}{c}{$(\mathrm{a})$} \\
\hline 0.036 & 0.045 & 0.027 & 0.031 \\
0.015 & 0.149 & 0.061 & 0.023 \\
0.007 & 0.094 & 0.156 & 0.034 \\
0.018 & 0.095 & 0.098 & 0.111 \\
\hline
\end{tabular}

(c) For Table 7(a)

\begin{tabular}{lcc}
\hline \multicolumn{1}{c}{$\lambda$} & $\Phi_{L S}^{(\lambda)}$ & $\gamma_{C L S}^{(\lambda)}$ \\
\hline 0 & 0.075 & 0.058 \\
0.5 & 0.094 & 0.073 \\
1 & 0.103 & 0.080 \\
\hline
\end{tabular}

\begin{tabular}{cccc}
\multicolumn{4}{c}{$(\mathrm{b})$} \\
\hline 0.036 & 0.027 & 0.045 & 0.031 \\
0.007 & 0.156 & 0.094 & 0.034 \\
0.015 & 0.061 & 0.149 & 0.023 \\
0.018 & 0.098 & 0.095 & 0.111 \\
\hline
\end{tabular}

(d) For Table 7(b)

\begin{tabular}{lcc}
\hline \multicolumn{1}{c}{$\lambda$} & $\Phi_{L S}^{(\lambda)}$ & $\gamma_{C L S}^{(\lambda)}$ \\
\hline 0 & 0.075 & 0.002 \\
0.5 & 0.094 & 0.002 \\
1 & 0.103 & 0.003 \\
\hline
\end{tabular}

\section{Concluding remarks}

For the square contingency table with ordered categories, we have considered a CLS model, which indicates the symmetry of cumulative probabilities for only one pair of symmetric cells. We have also proposed a measure to represent the degree of departure from the CLS model. The measure $\gamma_{C L S}^{(\lambda)}$ is useful for comparing the degrees of departure from CLS in different square tables (see Examples and Discussion). 


\section{Acknowledgements}

The authors would like to thank the referees for their comments.

\section{REFERENCES}

Agresti A. (2013): Categorical Data Analysis, 3rd Edition. John Wiley and Sons, Hoboken, New Jersey.

Bishop Y.M M., Fienberg S.E., Holland P.W. (1975): Discrete Multivariate Analysis: Theory and Practice. MIT Press, Cambridge.

Bowker A.H. (1948): A test for symmetry in contingency tables. Journal of the American Statistical Association 43: 572-574.

Patil G.P., Taillie C. (1982): Diversity as a concept and its measurement. Journal of the American Statistical Association 77: 548-561.

Saigusa Y., Tahata K., Tomizawa S. (2016): Measure of departure from partial symmetry for square contingency tables. Journal of Mathematics and Statistics 12: $152-156$.

Saigusa Y., Takami M., Ishii A., Nakagawa T., Tomizawa S. (2019a): Measure for departure from cumulative partial symmetry for square contingency tables with ordered categories. Journal of Statistics: Advances in Theory and Applications 21: 53-70.

Saigusa Y., Takami M., Ishii A., Tomizawa S. (2019b): Measure of departure from local symmetry for square contingency tables. International Journal of Statistics and Probability 8: 140-145.

Tomizawa S. (1994): Two kinds of measures of departure from symmetry in square contingency tables having nominal categories. Statistica Sinica 4: 325-334.

Tomizawa S., Seo T., Yamamoto H. (1998): Power-divergence-type measure of departure from symmetry for square contingency tables that have nominal categories. Journal of Applied Statistics 25: 387-398.

Tomizawa S., Miyamoto N., Hatanaka Y. (2001): Measure of asymmetry for square contingency tables having ordered categories. Australian and New Zealand Journal of Statistics 43: 335-349.

Tomizawa S., Miyamoto N., Iwamoto M. (2006): Linear column-parameter symmetry model for square contingency tables: application to decayed teeth data. Biometrical Letters 43: 91-98.

\section{APPENDIX}

Measures to represent the degree of departure from the S and PS models have been proposed (Tomizawa, 1994; Tomizawa et al., 1998; Saigusa et al., 2016), although the details are omitted here. Assuming that $p_{i j}+p_{j i} \neq 0$ for $i<j$ and $p_{k l} \neq p_{l k}$ for any $k<l$ except for any one pair $(k, l)$, Saigusa 
et al. (2019b) proposed a measure to represent the degree of departure from the LS model as follows:

$$
\Phi_{L S}^{(\lambda)}=\frac{\prod_{a=1}^{r-1} \prod_{b=a+1}^{r} \Phi_{a b}^{(\lambda)}}{\sum_{i=1}^{r-1} \sum_{j=i+1}^{r}\left[\left(p_{i j}^{*}+p_{j i}^{*}\right) \prod_{a=1}^{r-1} \prod_{\substack{b=a+1 \\(a, b) \neq(i, j)}}^{r} \Phi_{a b}^{(\lambda)}\right]} \text { for } \lambda>-1,
$$

where

$$
\begin{aligned}
& p_{i j}^{*}=\frac{p_{i j}}{\delta}, \quad \delta=\sum_{i=1}^{r} \sum_{\substack{j=1 \\
j \neq i}}^{r} p_{i j}, \quad p_{i j}^{c}=\frac{p_{i j}}{p_{i j}+p_{j i}}, \\
& \Phi_{i j}^{(\lambda)}= \begin{cases}1-\frac{\lambda 2^{\lambda}}{2^{\lambda}-1} I_{i j}^{(\lambda)} & \text { for } \lambda \neq 0, \\
1-\frac{1}{\log 2} I_{i j}^{(0)} & \text { for } \lambda=0,\end{cases} \\
& I_{i j}^{(\lambda)}= \begin{cases}\frac{1}{\lambda}\left(1-\left(p_{i j}^{c}\right)^{\lambda+1}-\left(p_{j i}^{c}\right)^{\lambda+1}\right) & \text { for } \lambda \neq 0, \\
-p_{i j}^{c} \log p_{i j}^{c}-p_{j i}^{c} \log p_{j i}^{c} & \text { for } \lambda=0 .\end{cases}
\end{aligned}
$$

Note that $I_{i j}^{(\lambda)}$ is the diversity index of degree $\lambda$, including the Shannon entropy when $\lambda=0$ (Patil and Taillie, 1982). When the LS model does not hold, we observe that

$$
\Phi_{L S}^{(\lambda)}=\frac{1}{\sum_{i=1}^{r-1} \sum_{j=i+1}^{r} \frac{p_{i j}^{*}+p_{j i}^{*}}{\Phi_{i j}^{(\lambda)}}} \quad \text { for } \lambda>-1
$$

Therefore, $\Phi_{L S}^{(\lambda)}$ is expressed as the weighted harmonic mean of the submeasure $\Phi_{i j}^{(\lambda)}$.

Assume that $G_{i j}+G_{j i} \neq 0$ for $i<j$. Tomizawa et al. (2001) proposed a measure for the $\mathrm{S}$ model as follows:

$$
\gamma_{S}^{(\lambda)}=\sum_{i=1}^{r-1} \sum_{j=i+1}^{r}\left(G_{i j}^{*}+G_{j i}^{*}\right) \gamma_{i j}^{(\lambda)} \quad \text { for } \lambda>-1,
$$


where

$$
\begin{aligned}
& G_{i j}^{*}=\frac{G_{i j}}{\Delta}, \quad \Delta=\sum_{i=1}^{r-1} \sum_{j=i+1}^{r}\left(G_{i j}+G_{j i}\right), \quad G_{i j}^{c}=\frac{G_{i j}}{G_{i j}+G_{j i}}, \\
& \gamma_{i j}^{(\lambda)}= \begin{cases}1-\frac{\lambda 2^{\lambda}}{2^{\lambda}-1} H_{i j}^{(\lambda)} & \text { for } \lambda \neq 0, \\
1-\frac{1}{\log 2} H_{i j}^{(0)} & \text { for } \lambda=0,\end{cases} \\
& H_{i j}^{(\lambda)}= \begin{cases}\frac{1}{\lambda}\left(1-\left(G_{i j}^{c}\right)^{\lambda+1}-\left(G_{j i}^{c}\right)^{\lambda+1}\right) & \text { for } \lambda \neq 0, \\
-G_{i j}^{c} \log G_{i j}^{c}-G_{j i}^{c} \log G_{j i}^{c} & \text { for } \lambda=0 .\end{cases}
\end{aligned}
$$

Saigusa et al. (2019a) proposed a measure for the CPS model as follows:

$$
\gamma_{C P S}^{(\lambda)}=\prod_{i=1}^{r-1} \prod_{j=i+1}^{r}\left[\gamma_{i j}^{(\lambda)}\right]^{\left(G_{i j}^{*}+G_{j i}^{*}\right)} \quad \text { for } \quad \lambda>-1 .
$$

It can be seen that the measures $\gamma_{S}^{(\lambda)}$ and $\gamma_{C P S}^{(\lambda)}$ are weighted arithmetic and geometric means of the submeasure $\gamma_{i j}^{(\lambda)}$, respectively. 